\title{
Developing a Hyperparameter Tuning Based Machine Learning Approach of Heart Disease Prediction
}

\author{
Emrana Kabir Hashi* and Md. Shahid Uz Zaman \\ Department of Computer Science \& Engineering, \\ Rajshahi University of Engineering \& Technology, Rajshahi, Bangladesh
}

\begin{abstract}
Machine learning techniques are widely used in healthcare sectors to predict fatal diseases. The objective of this research was to develop and compare the performance of the traditional system with the proposed system that predicts heart disease implementing the Logistic regression, K-nearest neighbor, Support vector machine, Decision tree, and Random Forest classification models. The proposed system helped to tune the hyperparameters using the grid search approach to the five mentioned classification algorithms. The performance of the heart disease prediction system is the major research issue. With the hyperparameter tuning model, it can be used to enhance the performance of the prediction models. The achievement of the traditional and proposed system was evaluated and compared in terms of accuracy, precision, recall, and F1 score. As the traditional system achieved accuracies between $81.97 \%$ and $90.16 \%$, the proposed hyperparameter tuning model achieved accuracies in the range increased between $85.25 \%$ and $91.80 \%$. These evaluations demonstrated that the proposed prediction approach is capable of achieving more accurate results compared with the traditional approach in predicting heart disease with the acquisition of feasible performance.
\end{abstract}

Keywords: Machine Learning, Heart Disease Prediction, Logistic regression, $K$ nearest neighbor, Support vector machine, Decision tree, Random Forest, Grid search

\section{Introduction}

Machine learning in the healthcare sector is an emerging topic to identify disease and diagnosis, discover drugs, and classify the medical image. The disease prediction system can be conducive for the hospital management, medical practitioners, doctors, physicians, nursing and residential care units, etc.

Every year the death rate of Cardiovascular Disease is increasing alarmingly. From the World Health Organization (WHO) report, it is found that for the year 2016 worldwide $31 \%$ of the deaths occurred due to cardiovascular disease [1-4]. In 2015, another report suggested that 17.7 million deaths have happened involved cardiovascular disease due to heart attack and stroke [4-7]. Early diagnosis and prediction of heart disease are more complicated when modern medical technology is not available. Many researchers try to develop a heart disease prediction model using different machine learning algorithms for the intervention of early treatment. For example, Logistic Regression (LR), K nearest neighbor (KNN), Support vector machine (SVM), Decision tree (DT), Random Forest (RF), Naïve Bayes (NB), and Artificial neural network (ANN) [1-20].

* Emrana Kabir Hashi. Tel.: +8801767616748

E-mail address: emranakabir@gmail.com

Manuscript History:

Received 07 October, 2020, Revised 28 October, 2020, Accepted 28 October, 2020, Published 30 October, 2020

e-ISSN: 2289-7771

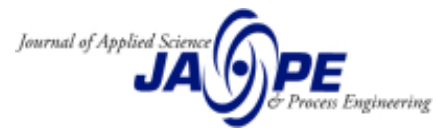


Healthcare Sector is one of the most important sectors where people expect the highest level of treatment facilities and services. So, the motivation of this work is to improve the performance of the machine learning algorithms by performing a grid search. After applying the grid search, the optimal parameters of the machine learning algorithms can be selected. Using these tuned hyperparameters, the performance of the heart disease prediction system can be enhanced.

The contributions of research work are stated in the following.

- In the first phase, the authors developed a traditional heart disease prediction model using Logistic Regression (LR), K nearest neighbor (KNN), Support vector machine (SVM), Decision tree (DT), Random Forest (RF) algorithms.

- In the second phase, the authors proposed a prediction system where five machine algorithms are applied with a hyperparameter tuning approach. Here, a Grid search is applied to find the optimal hyperparameters of each algorithm.

- Finally, the performance of these two systems such as accuracy, precision, recall, and F1 score has been compared by the standard state.

\section{Literature Review}

This section mainly summarizes the contributions of existing heart disease prediction approaches. Researchers have developed many machine learning classification models to predict heart disease dataset.

The research work [1] developed a proposed system that solved the feature selection problem employing a fast conditional mutual information feature selection algorithm. This model applied with Support Vector Machine classifier and achieved an accuracy was $92.37 \%$. The paper [2] introduced a system that stacks two SVM models for the effective prediction of heart disease and achieved accuracies in the range of $57.85 \%$ and $91.83 \%$. The paper [3] used different machine learning classifiers including the decision tree, random forest, SVM, neural networks, and LR. This was obtained that SVM was the best classifier model with $\mathrm{AUC}=0.75$. For the prediction of the heart disease dataset, the paper [4] proposed a framework that was executed using five algorithms RF, Naïve Bayes, SVM, Hoeffding DT, and Logistic Model Tree. After selecting the best features, these algorithms correctly classified heart disease with accuracies between $81.24 \%$ and $95.05 \%$. The study [5] presented a hyperparameter tuning model using a DT and experimented with 102 heterogeneous datasets. The work [6] used DT, SVM, $\mathrm{RF}$, and LR to build the prediction models and the RF has achieved the best accuracy at $90 \%$. The research [7] proposed a fine-tune prediction model to identify significant features and also build a classification model including RF, SVM, and DT model to achieve high predictive accuracy. The paper [8] presented a study that ensemble techniques, such as bagging and boosting, were effective in improving the prediction accuracy (85.48\%). The review paper [9] described various research works of machine learning in the prediction of heart diseases. The paper [10] proposed a system and compared DT, SVM, RF, and LR with the selected features as well as full features. In the review paper [11], from 1992 to 2019 all relevant studies based on heart disease diagnosis were summarized. The paper [12] experimented with k-NN, DT, Naive Bayes, LR, SVM, Neural Network to predict cardiovascular disease. The research [13] developed a system with DT, LR, SVM, Naïve Bayes, and RF classifiers to select optimal features and improve accuracy. The research paper [14] produced an enhanced performance level with an accuracy level of $88: 7 \%$ through the prediction model for heart disease with the hybrid random forest with a linear model. The research [16] developed a heart disease predictive system with the DT, LR, SVM, MLP, Naïve Bayes, and RF classifiers to select optimal features and improve accuracy. The study [17] presented a model to predict the classification model and to know which selected features play a key role in the prediction of heart disease by using Cleveland and statlog project heart datasets. The study [18] developed a method of classifying for heart disease degree of patient-based characteristic data using an adaptive neuro-fuzzy inference system. The paper [19] proposed a hybrid intelligent system framework for the prediction of heart disease using different

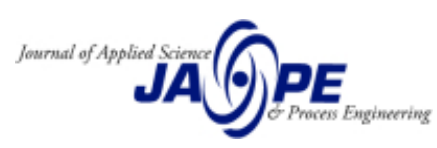


machine learning classifiers. In the paper [20], the authors proposed a heart prediction model where the noise was detected and eliminated. The study developed XGBoost to predict heart disease.

The researchers have been developed a heart disease prediction system without hyperparameters tuning. So, we have proposed a heart disease prediction system using different machine learning algorithms with hyperparameters tuning approach.

\section{Materials and Methods}

In this section, the research materials and methodologies are presented and discussed in brief.

\subsection{Dataset Description}

In this research work, the Cleveland Heart Disease dataset [1-3] has been collected from the UCI machine learning repository that has used for both training and testing purposes. It contains 303 instances and 75 attributes, but this work considers a feature subset of 14 numerical valued attributes. The output level has two classes, where 0 represents not having heart disease, and 1 represents having heart disease. The information on the heart disease dataset is given in Table 1. Where, the attribute name, description, minimum, maximum, mean, and standard deviation are presented.

Table 1. Heart disease dataset description

\begin{tabular}{|c|c|c|c|c|c|}
\hline $\begin{array}{l}\text { Attribute } \\
\text { Name }\end{array}$ & Attribute Description & mean & std & $\min$ & $\max$ \\
\hline age & age in years & 54.37 & 9.08 & 29.00 & 77.00 \\
\hline $\operatorname{sex}$ & 1: male, 2 : female & 0.68 & 0.47 & 0.00 & 3.00 \\
\hline Chest pain & $\begin{array}{c}\text { 1: typical angina, } 2 \text { : typical type angina, } 3: \text { non- } \\
\text { angina pain, } 4 \text { : asymptomatic }\end{array}$ & 0.97 & 1.03 & 0.0 & 3.0 \\
\hline thestbps & resting blood pressure (in $\mathrm{mm} \mathrm{Hg}$ ) & 131.62 & 17.54 & 94.0 & 200.0 \\
\hline chol & serum cholestoral in $\mathrm{mg} / \mathrm{dl}$ & 246.26 & 51.83 & 126.0 & 564.0 \\
\hline fbs & fasting blood sugar $>120 \mathrm{mg} / \mathrm{dl}), 1=$ true; $0=$ false & 0.15 & 0.36 & 0.0 & 1.0 \\
\hline restecg & $\begin{array}{c}0: \text { Nothing to note } \\
\text { 1: ST-T Wave abnormality } \\
\text { 2: Possible or definite left ventricular hypertrophy }\end{array}$ & 0.53 & 0.53 & 0.0 & 2.0 \\
\hline thalach & maximum heart rate achieved & 149.65 & 22.91 & 71.0 & 202.0 \\
\hline exang & exercise induced angina $(1=$ yes; $0=$ no $)$ & 0.33 & 0.47 & 0.0 & 1.0 \\
\hline oldpeak & ST depression : continuous value & 1.04 & 1.16. & 0.0 & 6020 \\
\hline solpe & $\begin{array}{l}\text { 0: Upsloping: better heart rate with exercise } \\
\text { 1: Flatsloping: minimal change } \\
\text { 2: Downslopins: signs of unhealthy heart }\end{array}$ & 1.40 & 1.16 & 0.0 & 6.20 \\
\hline $\mathrm{ca}$ & $\begin{array}{c}\text { number of major vessels (0-3) colored by } \\
\text { flourosopy }\end{array}$ & 0.73 & 1.02 & 0.0 & 4.0 \\
\hline thal & $\begin{array}{c}\text { 1,3: normal } \\
\text { 6: fixed defect: used to be defect but ok now } \\
\text { 7: reversible defect: no proper blood movement } \\
\text { when exercising }\end{array}$ & 2.31 & 0.61 & 0.0 & 3.0 \\
\hline target & \multicolumn{5}{|c|}{ Heart disease patient $=1$, healthy $=0$} \\
\hline
\end{tabular}




\subsection{Methodology}

The research has considered two stages of heart disease prediction. The traditional heart disease prediction system has presented without a hyperparameter tuning approach of the machine learning algorithm, and the block diagram has shown in Figure 1.

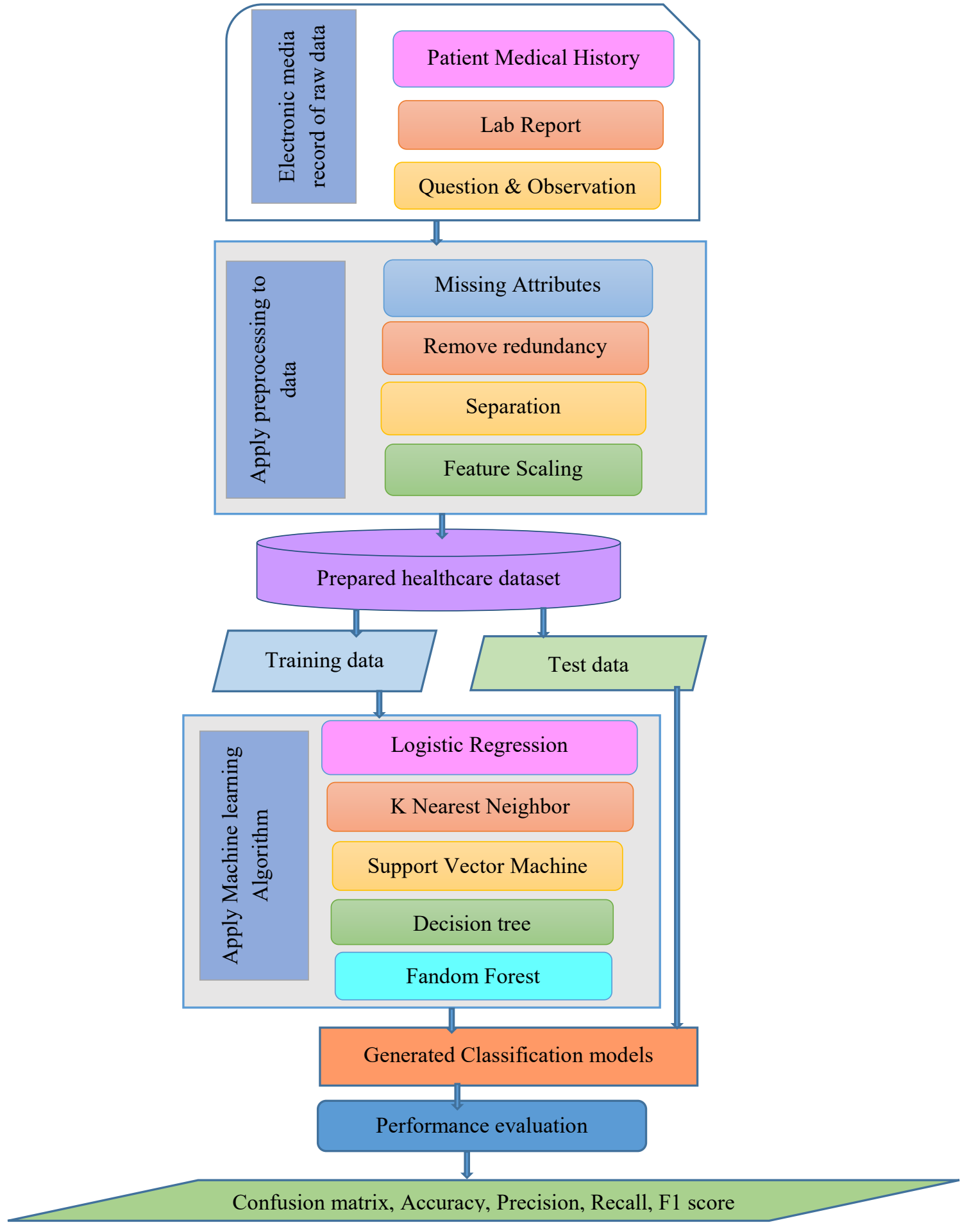

Figure 1: Block diagram of the traditional system 
This research has proposed a prediction system with a hyperparameter tuning approach of the machine learning algorithm, and the block diagram has presented in Figure 2.

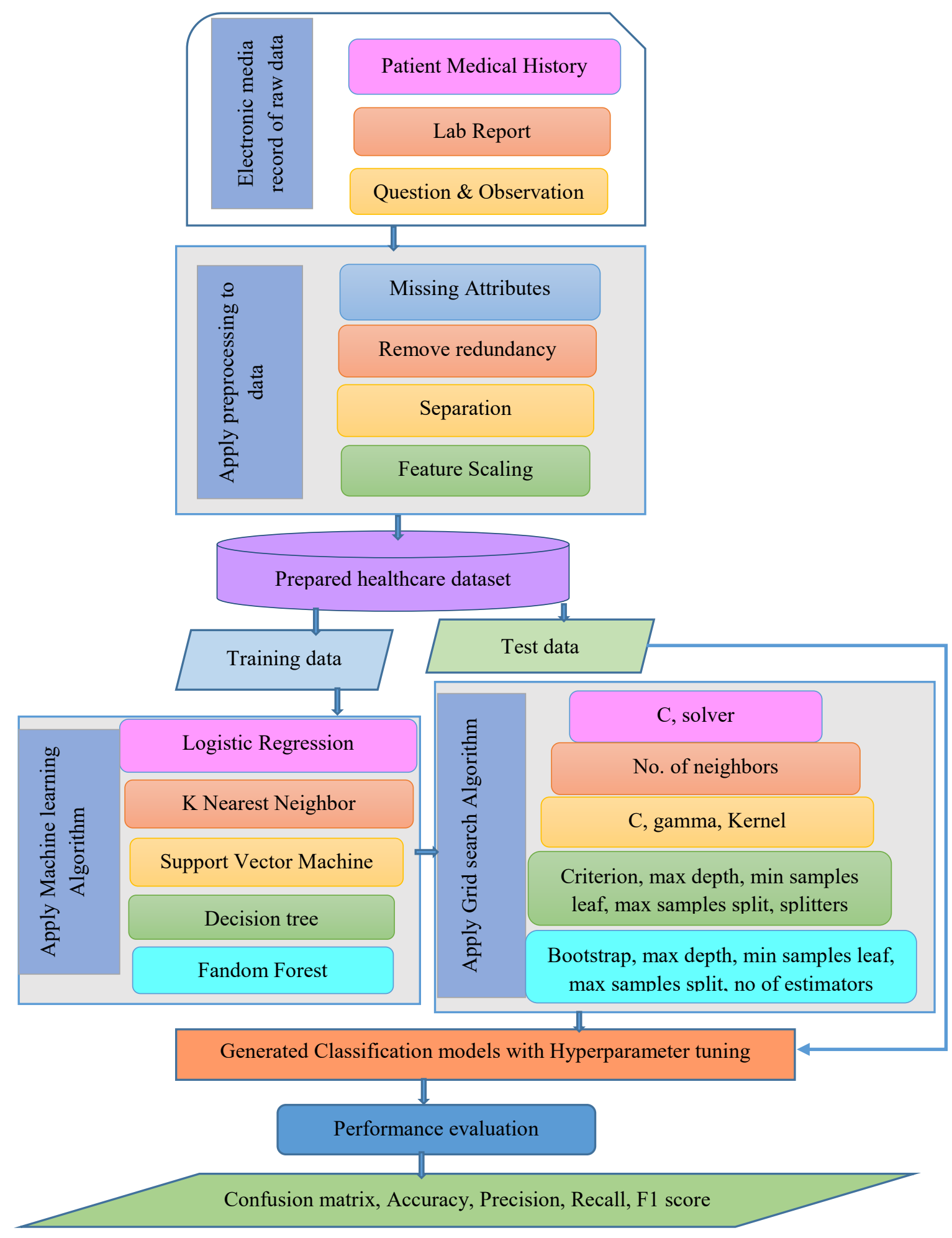

Figure 2. Block diagram of the proposed system 


\subsubsection{Data collection}

Medical data are collected from different sources such as patient medical history, laboratory reports, questions, and observations which are stored as text, a numeric value, or image format. After collecting data, many researchers can use this electronic media raw data to experiment with different healthcare prediction models. In this research work, the heart disease dataset was collected from the UCI machine learning repository.

\subsubsection{Data preprocessing}

In this step data preprocessing is applied to identify the missing values, to process the noisy, incomplete, irreverent, and inconsistent value, to remove the redundancy of some attributes. Then separation, feature scaling, and normalization are performed to find the standard formate of data. After data preparation, the dataset is divided into a training set ( $80 \%$ of data) and a test set ( $20 \%$ of data).

\subsubsection{Model generation}

In the stage, machine learning algorithms are applied to the training set to develop different classification models. After that test set, individual samples are classified based on the generated models. In Figure 1 and Figure 2, both traditional and proposed models are developed with the use of five machine learning algorithms named LR, KNN, SVM, DT, and RF classifiers. Then using this five generated model, the test set is classified and evaluated the performance. In figure 1, the traditional system is performed without any hyperparameter tuning method. In this system, the default parameters are used to generate these five classification models.

In Figure 2, the proposed system is performed with the help of grid search, and the crossvalidation approaches the hyperparameters are optimized and tuned. In machine learning, hyperparameter tuning is one of the most significant research issues. If the hyperparameters are tuned or optimized then it is considered that the machine learning algorithms can give better performance. Grid search is the traditional approach that is used to tune the optimal parameters for many machine learning algorithms. It considers cross-validation to guide the performance metrics. Grid search is an exhaustive search which can exercise to compute the optimal values of hyperparameters. It can build a model that generates every parameter combination and also stores each combination of the model. The efforts and resources can be saved using this search. Then with the tuned parameters, the LR, KNN, SVM, DT, and RF classifier models are generated. After the generation of the classification model, the test set is applied to the proposed model with the tune hyperparameter and evaluated the performance of the test set.

\subsubsection{Machine learning algorithms}

In the model generation process, Logistic regression (LR), K nearest neighbor (KNN), Support vector machine (SVM), Decision tree (DT), and Random Forest (RF) classifiers are used as machine learning algorithms. These five algorithms are selected for the heart disease prediction system because these algorithms perform better than other machine learning algorithms.

\subsubsection{Logistic regression}

Logistic regression is a machine learning technique from the field of statistics. It is mainly used for a binary classification problem and uses a logistic function to predict a binary dependent variable [1] [6] [11] [16].

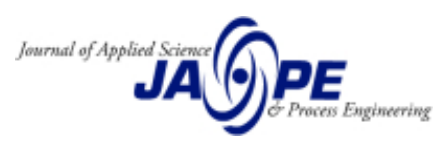




\subsubsection{K nearest neighbor}

$\mathrm{KNN}$ is used widely in the machine learning classification problem. It is simple to understand and generates a non-parametric model that is applied to practical problems. It is a lazy learner or instancebased learner which depend on the distance. It works well but does not learn any classification rule.

\subsubsection{Support vector machine}

SVM is a supervised machine learning algorithm that is classified instances by finding an optimal hyperplane that separates the classes on different dimensional spaces [1] [6] [11] [16]. It can build a knowledge-based model using the linear kernel, radial basis function (RBF) kernel, sigmoid and polynomial kernel.

\subsubsection{Decision tree}

DT is a supervised machine learning algorithm that generates a tree structure to predict the target class. In the tree model, leaf nodes are called decision nodes and internal nodes represent features and each branch represents the outcome of the test. The features are selected by calculating entropy and information gain [1] [6] [11] [16].

\subsubsection{Random Forest}

$\mathrm{RF}$ is a supervised machine learning algorithm that is widely used for both classification and regression problems. It creates decision trees on randomly selected training datasets, gets the prediction from the collection of trees, and casts a unit vote for the most popular class to classify an input vector $[4][6][11]$.

\subsubsection{Performance Evaluation}

In this step, both the systems evaluate the performance of the training set and test set and find the confusion matrix. Then the performance metrics of these two models have been calculated and evaluated in terms of accuracy, precision, recall, and F1 score with the help of the confusion matrix. Accuracy is the ratio of correctly classified observations to the total number of observations. Precision is performed by taking the ratio of correctly classified positive samples to the total predicted positive samples. The recall is calculated by taking the ratio of truly classified positive samples to all samples in actual class yes. F1 score is performed by taking the weighted average of precision and recall [1][2]. The mathematical expressions of accuracy, precision, recall, and F1 score are shown in the equation (1), (2), (3), and (4) respectively.

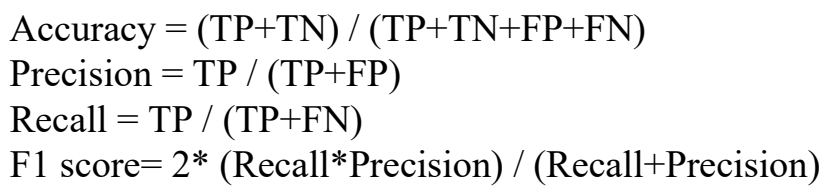

Where, TP, FN, FP, and TN represented as True Positive, False Negative, False Positive, and True Negative, respectively. 


\section{Experimental Results Analysis and Discussion}

\subsection{Result of data preprocessing}

The heart disease dataset consists of 303 samples with 14 attributes where 138 instances having heart disease and 165 are healthy instances. In the preprocessing step, the statistical operation has been performed to identify and remove the missing values and to find the maximum, minimum, mean, and standard deviation of each feature set.
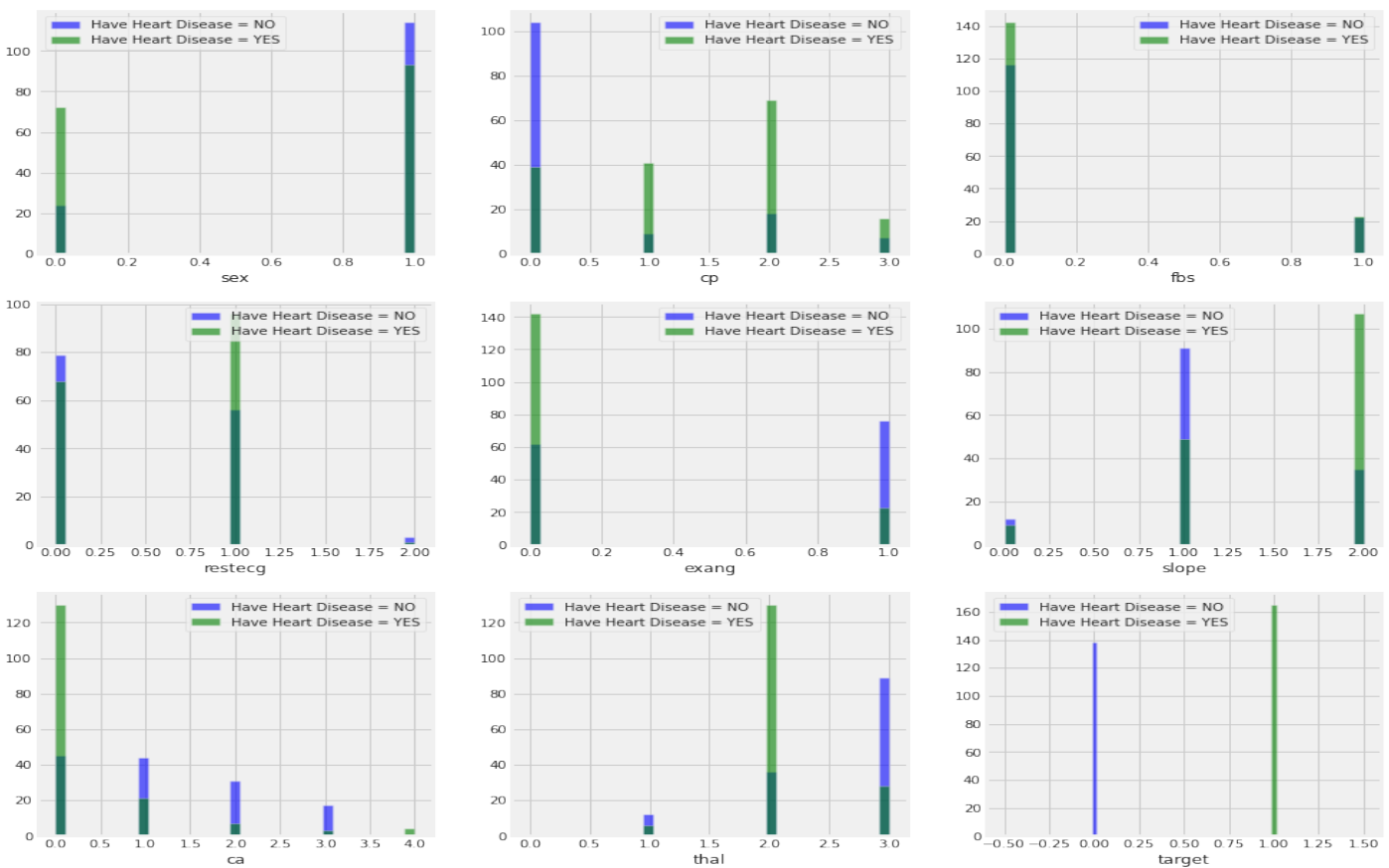

Figure 3. Histogram of categorical valued attributes.
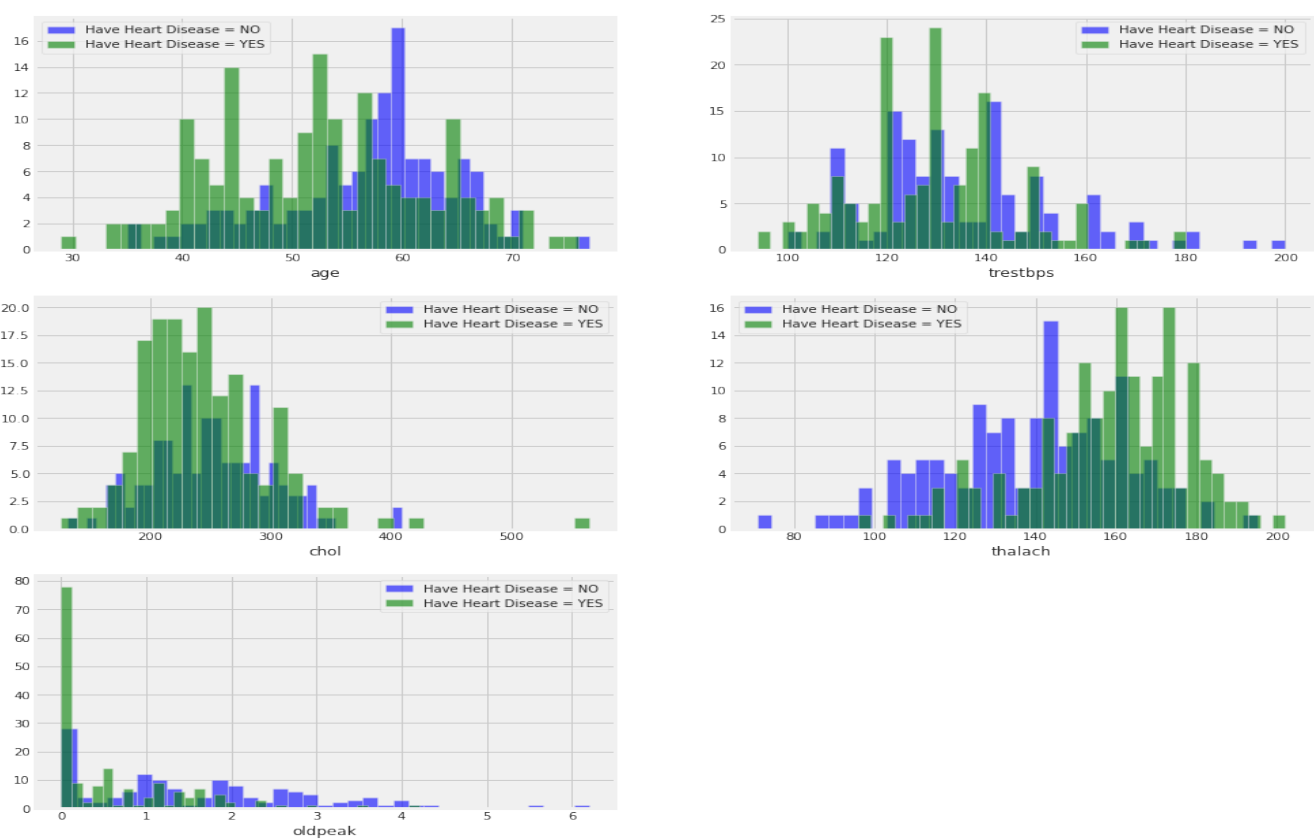

Figure 4. Histogram of continuous-valued attributes. 
Then the histogram of categorical and continuous features has been plotted for easy and better understanding. The histogram plots are presented for the pattern and frequency distribution of continuous and categorical measurements of data. The distribution of each feature value is shown in Figure 3 and Figure 4 as a histogram plot. It can help to identify the trend and patterns of data to understand the distribution of features.

Figure 5 represents the heat map which describes the co-relation among the features of the heart disease dataset. Here, different colors have been used to represent the values on the two-dimensional surface. It can be visualized that categorical valued attributes are more concentrated than the continuous-valued attributes. The heat map of the heart disease dataset has depicted for the hierarchical clustering and general view of numeric data.

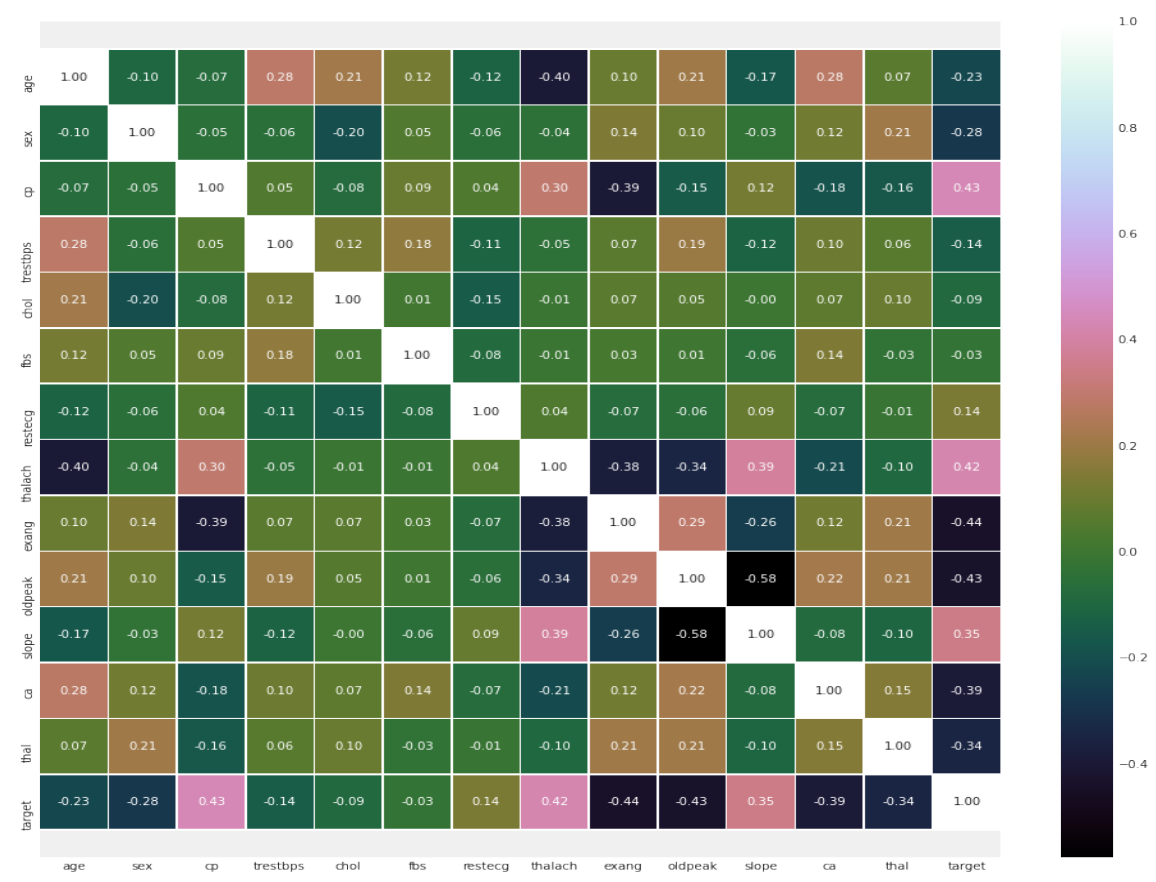

Figure 5. The heat map for correlation features of the heart disease dataset.

After investigating the dataset, the categorical valued attributes have been converted into dummy attributes. Then, centering and scaling operation has been performed to standardize each feature by computing the relevant statistics on the dataset. The resultant dataset has been divided into a training set and a test set.

\subsection{Experimental results of the traditional and proposed system}

The experimental results of different classifiers of the traditional system and proposed system are given in the following sections.

\subsubsection{Performance evaluation and comparison of the traditional system}

In this experiment, the machine learning algorithms are applied with the default parameters. Table 2 shows the result of this system.

In the training phase, Logistic Regression is fitted and executed the model with parameters of $\mathrm{C}=1$ and solver $=$ 'liblinear' and found $87.60 \%, 87.05 \%, 90.98 \%$, and $88.97 \%$ of accuracy, precision, 
recall, and F1 score respectively. The test set is predicted on this LR model and provides $88.52 \%$, $90.32 \%, 87.50 \%$, and $88.89 \%$ of accuracy, precision, recall, and F1 score respectively.

Again in the training phase, $\mathrm{KNN}$ is fitted and executed the model with the parameters of no. of neighbor $=5$ and weights = 'uniform' and found $87.60 \%, 87.59 \%, 90.23 \%$, and $88.89 \%$ of accuracy, precision, recall, and F1 score respectively. The test set is predicted on this KNN model and provides $90.16 \%, 90.62 \%, 90.62 \%$, and $90.62 \%$ of accuracy, precision, recall, and F1 score respectively.

Table 2. Performance of evaluation and comparison of classification models on the training set and the test set.

\begin{tabular}{|c|c|c|c|c|c|c|c|c|c|}
\hline \multicolumn{2}{|c|}{ Traditional system } & \multicolumn{4}{|c|}{$\begin{array}{c}\text { Performance evaluation of Training } \\
\text { dataset }\end{array}$} & \multicolumn{4}{|c|}{ Performance evaluation of Test dataset } \\
\hline $\begin{array}{c}\text { Machine } \\
\text { learning } \\
\text { algorithms }\end{array}$ & Parameters & $\begin{array}{l}\text { Accur } \\
\text { acy } \\
(\%)\end{array}$ & $\begin{array}{c}\text { Precision } \\
\text { (\%) }\end{array}$ & $\begin{array}{c}\text { Recall } \\
(\%)\end{array}$ & $\begin{array}{c}\text { F1 } \\
\text { score } \\
(\%)\end{array}$ & $\begin{array}{l}\text { Accura } \\
\text { cy (\%) }\end{array}$ & $\begin{array}{c}\text { Precision } \\
\text { (\%) }\end{array}$ & $\begin{array}{c}\text { Recall } \\
(\%)\end{array}$ & $\begin{array}{c}\text { F1 } \\
\text { scor } \\
\text { e } \\
(\%)\end{array}$ \\
\hline LR & $\begin{array}{c}\mathrm{C}=1 \\
\text { solver= 'liblinear' }\end{array}$ & 87.60 & 87.05 & 90.98 & 88.97 & 88.52 & 90.32 & 87.50 & $\begin{array}{c}88.8 \\
9\end{array}$ \\
\hline KNN & $\begin{array}{l}\text { No of neighbor }=5 \\
\text { weights = 'uniform' }\end{array}$ & 87.60 & 87.59 & 90.23 & 88.89 & 90.16 & 90.62 & 90.62 & $\begin{array}{c}90.6 \\
2\end{array}$ \\
\hline SVM & $\begin{array}{c}\text { kernel }=\text { 'rbf' } \\
\text { gamma }=0.001 \\
\mathrm{C}=2.0\end{array}$ & 81.82 & 77.30 & 94.74 & 85.14 & 88.52 & 87.88 & 90.62 & $\begin{array}{c}89.2 \\
3\end{array}$ \\
\hline DT & $\begin{array}{c}\text { criterion= 'gini' } \\
\text { min samples leaf= } \\
1 \\
\text { min samples split }= \\
2 \\
\text { splitter }=\text { best }\end{array}$ & 100 & 100 & 100 & 100 & 81.97 & 86.21 & 78.12 & $\begin{array}{c}81.9 \\
7\end{array}$ \\
\hline $\mathbf{R F}$ & $\begin{array}{c}\text { criterion }=\text { 'gini' } \\
\text { min samples leaf }= \\
1 \text { min samples } \\
\text { split }=2 \\
\text { no of estimators= } \\
1000\end{array}$ & 100 & 100 & 100 & 100 & 85.25 & 87.10 & 84.38 & $\begin{array}{c}85.7 \\
1\end{array}$ \\
\hline
\end{tabular}

In another training model, SVM is fitted and executed the model with the parameters of $\mathrm{C}=2.0$, gamma $=0.001$, and RBF kernel, and found $81.82 \%, 77.30 \%, 94.74 \%$, and $85.14 \%$ of accuracy, precision, recall, and F1 score respectively. The test set is predicted on this SVM model and provides $88.52 \%, 87.88 \%, 90.62 \%$, and $89.23 \%$ of accuracy, precision, recall, and F1 score, respectively.

In the fourth phase of training, DT is fitted and executed the model with the parameters of best splitter and Gini index and found $100 \%, 100 \%, 100 \%$, and $100 \%$ of accuracy, precision, recall, and F1 score respectively. The test set is predicted on this DT model and provides $81.97 \%, 86.21 \%, 78.12 \%$, and $81.97 \%$ of accuracy, precision, recall, and F1 score, respectively.

In the last phase of training, RF is fitted and executed the model with the parameters of 1000 no of estimators and Gini index and found $100 \%, 100 \%, 100 \%$, and $100 \%$ of accuracy, precision, recall, and F1 score respectively. The test set is predicted on this RF model and provides $85.25 \%, 87.10 \%$, $84.38 \%$, and $85.71 \%$ of accuracy, precision, recall, and F1 score, respectively.

In Figure 6, the training and testing performance based on the classifiers of the traditional system is graphically analyzed. 


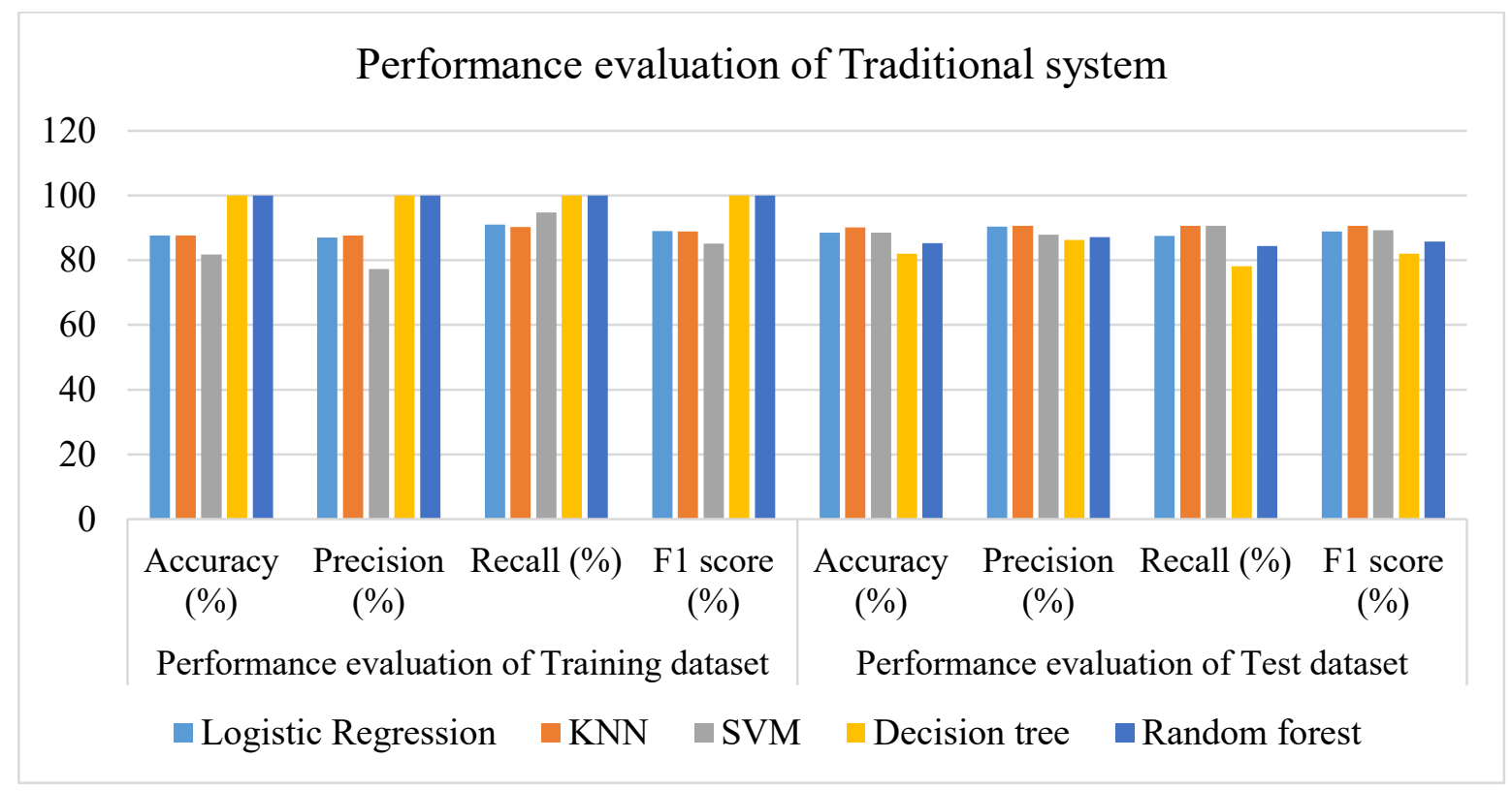

Figure 6. The graphical analysis of Performance evaluation of Traditional system

\subsubsection{Performance evaluation and comparison of the proposed system}

In the proposed system, the Grid search is used to find the optimal hyperparameters. After tuning the hyperparameters, the classification models are generated. Table 3 shows the result of the proposed system.

In the training phase, LR is fitted and executed with the tuned hyperparameters of $\mathrm{C}=0.23$ and solver $=$ 'liblinear' and found $85.54 \%, 85.0 \%, 89.47 \%$, and $87.18 \%$ of accuracy, precision, recall, and F1 score respectively. The test set is predicted on this LR model and provides $90.16 \%, 93.33 \%, 87.50 \%$, and $90.32 \%$ of accuracy, precision, recall, and F1 score, respectively. Again in the training phase, KNN is fitted and executed with the tuned hyperparameters of no. of neighbor $=8$ and weights $=$ 'uniform' and found $85.95 \%, 87.22 \%, 87.22 \%$, and $87.22 \%$ of accuracy, precision, recall, and F1 score respectively. The test set is predicted on this KNN model and provides $91.80 \%, 93.55 \%, 90.62 \%$, and $90.06 \%$ of accuracy, precision, recall, and F1 score respectively. In another training model, SVM is fitted and executed with the tuned hyperparameters of $\mathrm{C}=1.0$, gamma $=0.1$, and $\mathrm{RBF}$ kernel, and found $92.56 \%$, $91.97 \%, 94.74 \%$, and $93.33 \%$ of accuracy, precision, recall, and F1 score respectively. The test set is predicted on this SVM model and provides $90.16 \%, 93.33 \%, 87.50 \%$, and $90.32 \%$ accuracy, precision, recall, and F1 score, respectively. In the fourth phase of training, DT is fitted and executed with the tuned hyperparameters of a random splitter, entropy, 13 minimum samples of the leaf, and 2 minimum samples the split, maximum depth $=3$ and found $84.71 \%, 82.88 \%, 90.98 \%$, and $86.74 \%$ of accuracy, precision, recall, and F1 score respectively. The test set is predicted on this DT model and provides $86.89 \%, 85.29 \%, 90.62 \%$, and $87.88 \%$ of accuracy, precision, recall, and F1 score respectively. In the last phase of training, RF is fitted and executed with the tuned hyperparameters of 200 no of estimators, Gini index, 1 minimum sample of the leaf, and 2 minimum samples the split, maximum depth $=3$, maximum features $=$ square, and found $100 \%, 100 \%, 100 \%$, and $100 \%$ of accuracy, precision, recall, and F1 score respectively. The test set is predicted on this RF model and provides $85.25 \%, 87.10 \%$, $84.38 \%$, and $85.71 \%$ of accuracy, precision, recall, and F1 score, respectively.

In Figure 7, the training and testing performance based on the classifiers of the proposed system is graphically analyzed.

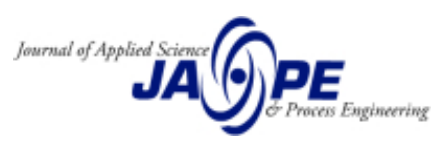


Table 3. Performance of evaluation and comparison of classification models with a hyperparameter tuning approach on the training set and the test set.

\begin{tabular}{|c|c|c|c|c|c|c|c|c|c|}
\hline \multicolumn{2}{|c|}{$\begin{array}{c}\text { The proposed system with } \\
\text { hyperparameter tuning }\end{array}$} & \multicolumn{4}{|c|}{$\begin{array}{c}\text { Performance evaluation of } \\
\text { Training dataset }\end{array}$} & \multicolumn{4}{|c|}{$\begin{array}{c}\text { Performance evaluation of } \\
\text { Test dataset }\end{array}$} \\
\hline $\begin{array}{l}\text { Machine } \\
\text { learning } \\
\text { algorithms }\end{array}$ & Tuned hyperparameters & $\begin{array}{c}\text { Accuracy } \\
(\%)\end{array}$ & $\begin{array}{l}\text { Precision } \\
(\%)\end{array}$ & $\begin{array}{c}\text { Recall } \\
(\%)\end{array}$ & $\begin{array}{c}\text { F1 } \\
\text { score } \\
(\%)\end{array}$ & $\begin{array}{c}\text { Accuracy } \\
(\%)\end{array}$ & $\begin{array}{l}\text { Precision } \\
(\%)\end{array}$ & $\begin{array}{c}\text { Recall } \\
(\%)\end{array}$ & $\begin{array}{c}\text { F1 } \\
\text { score } \\
\text { (\%) }\end{array}$ \\
\hline LR & $\begin{aligned} \mathrm{C} & =0.23 \\
\text { solver } & =\text { 'liblinear' }\end{aligned}$ & 85.54 & 85.00 & 89.47 & 87.18 & 90.16 & 93.33 & 87.50 & 90.32 \\
\hline KNN & $\begin{array}{l}\text { No of neighbor }=8 \\
\text { weights= 'uniform' }\end{array}$ & 85.95 & 87.22 & 87.22 & 87.22 & 91.80 & 93.55 & 90.62 & 92.06 \\
\hline SVM & $\begin{array}{c}\text { kernel }=\text { 'rbf' } \\
\text { gamma }=0.1, C=1.0\end{array}$ & 92.56 & 91.97 & 94.74 & 93.33 & 90.16 & 93.33 & 87.50 & 90.32 \\
\hline DT & $\begin{array}{c}\text { criterion= 'entropy' } \\
\text { min samples leaf }=13 \\
\text { min samples split= } 2 \\
\text { max_depth=3 } \\
\text { splitter= 'random' }\end{array}$ & 84.71 & 82.88 & 90.98 & 86.74 & 86.89 & 85.29 & 90.62 & 87.88 \\
\hline $\mathbf{R F}$ & $\begin{array}{c}\text { criterion= 'gini', } \\
\text { min samples leaf= } 1 \\
\text { min samples split= } 2 \\
\text { no of estimators= } 200 \\
\text { max_features= 'sqrt' } \\
\text { max_depth }=50\end{array}$ & 100 & 100 & 100 & 100 & 85.25 & 87.10 & 84.38 & 85.71 \\
\hline
\end{tabular}

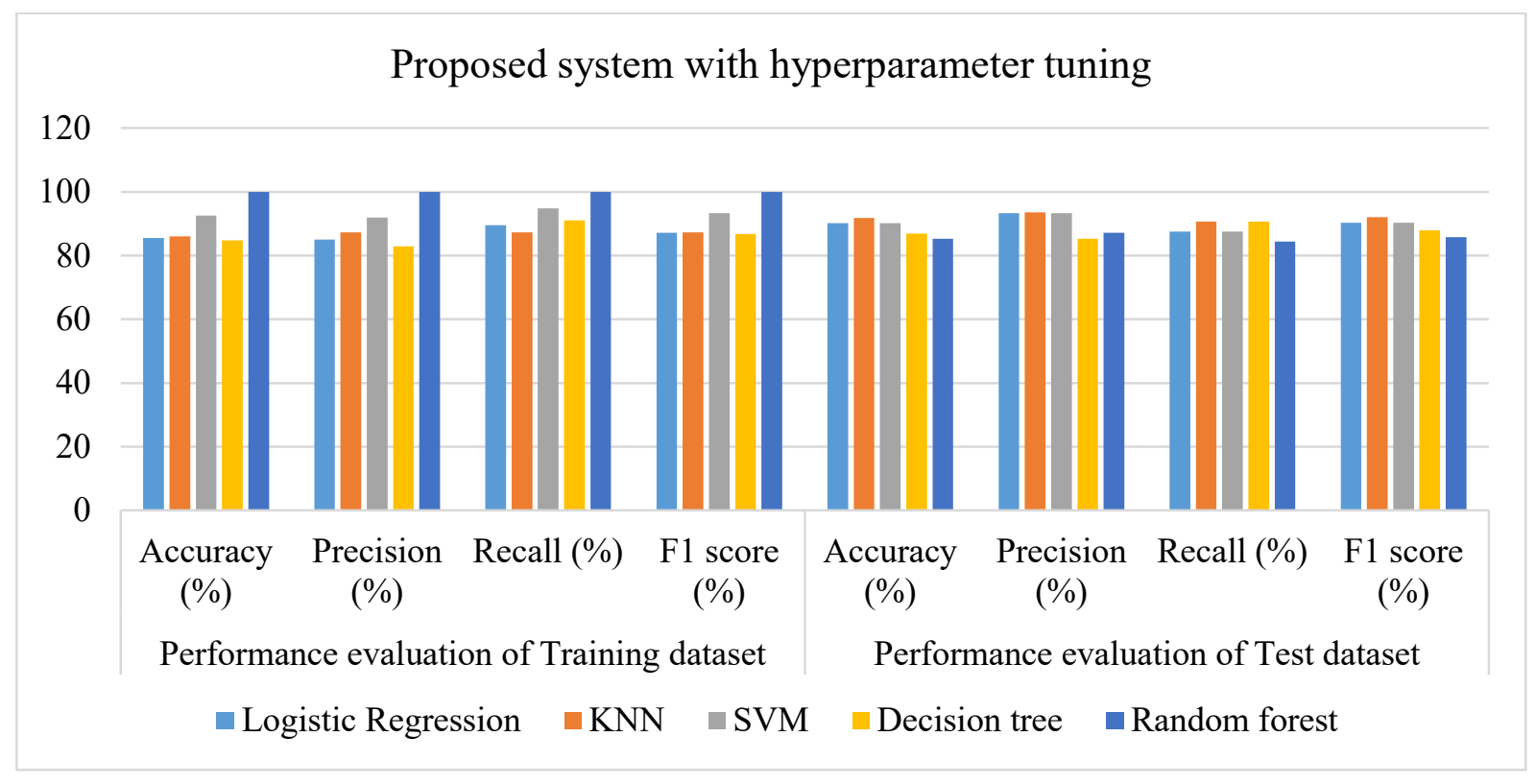

Figure 7: The graphical analysis of Performance evaluation of proposed system 


\subsubsection{Performance comparison of the proposed system with the traditional system}

Table 4, Table 5, Table 6, and Table 7 describe the performance comparison of the proposed system with the traditional system in terms of accuracy, precision, recall, and F1 score, respectively. Figure 8, Figure 9, Figure 10, and Figure 11 show the graphical comparison of performances.

Table 4. Comparison of accuracy

\begin{tabular}{|c|c|c|c|c|}
\hline \multirow{2}{*}{$\begin{array}{c}\text { Machine } \\
\text { learning } \\
\text { algorithms }\end{array}$} & \multicolumn{2}{|c|}{ Accuracy (\%) of Training dataset } & \multicolumn{2}{|c|}{ Accuracy (\%) of Test dataset } \\
\hline & $\begin{array}{l}\text { Without } \\
\text { parameter } \\
\text { tuning }\end{array}$ & $\begin{array}{c}\text { With } \\
\text { Hyperparameter } \\
\text { tuning }\end{array}$ & $\begin{array}{l}\text { Without } \\
\text { parameter } \\
\text { tuning }\end{array}$ & $\begin{array}{c}\text { With } \\
\text { Hyperparameter } \\
\text { tuning }\end{array}$ \\
\hline $\mathbf{L R}$ & 87.60 & 85.54 & 88.52 & 90.16 \\
\hline KNN & 87.60 & 85.95 & 90.16 & 91.80 \\
\hline SVM & 81.82 & 92.56 & 88.52 & 90.16 \\
\hline DT & 100 & 84.71 & 81.97 & 86.89 \\
\hline $\mathbf{R F}$ & 100 & 100 & 85.25 & 85.25 \\
\hline
\end{tabular}

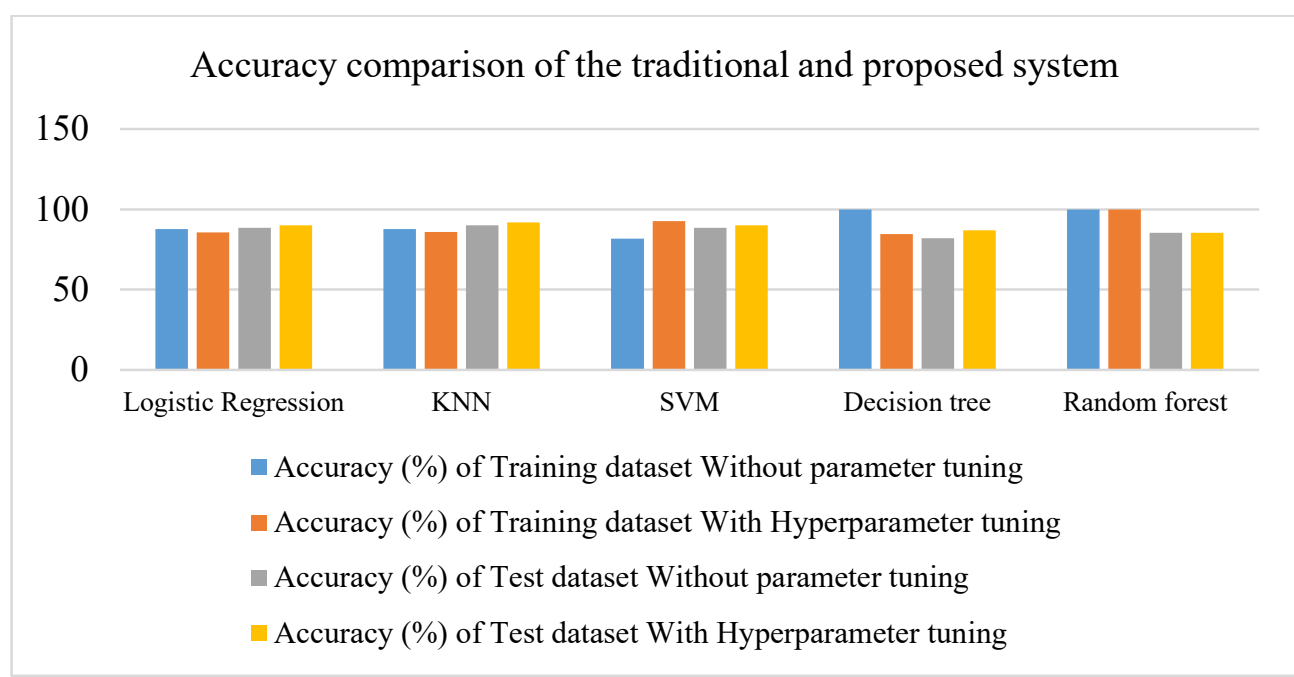

Figure 8. The graphical comparison of accuracy.

Table 5. Comparison of precision

\begin{tabular}{|c|c|c|c|c|}
\hline $\begin{array}{c}\text { Machine } \\
\text { learning } \\
\text { algorithms }\end{array}$ & \multicolumn{2}{|c|}{ Precision (\%) of Training dataset } & \multicolumn{2}{|c|}{ Precision (\%) of Test dataset } \\
\cline { 2 - 5 } & $\begin{array}{c}\text { Without } \\
\text { parameter } \\
\text { tuning }\end{array}$ & $\begin{array}{c}\text { With } \\
\text { Hyperparameter } \\
\text { tuning }\end{array}$ & $\begin{array}{c}\text { Without } \\
\text { parameter } \\
\text { tuning }\end{array}$ & $\begin{array}{c}\text { With } \\
\text { Hyperparameter } \\
\text { tuning }\end{array}$ \\
\hline LR & 87.05 & 85.00 & 90.32 & 93.33 \\
\hline KNN & 87.59 & 87.22 & 90.62 & 93.55 \\
\hline SVM & 77.30 & 91.97 & 87.88 & 93.33 \\
\hline DT & 100 & 82.88 & 86.21 & 85.29 \\
\hline RF & 100 & 100 & 87.10 & 87.10 \\
\hline
\end{tabular}




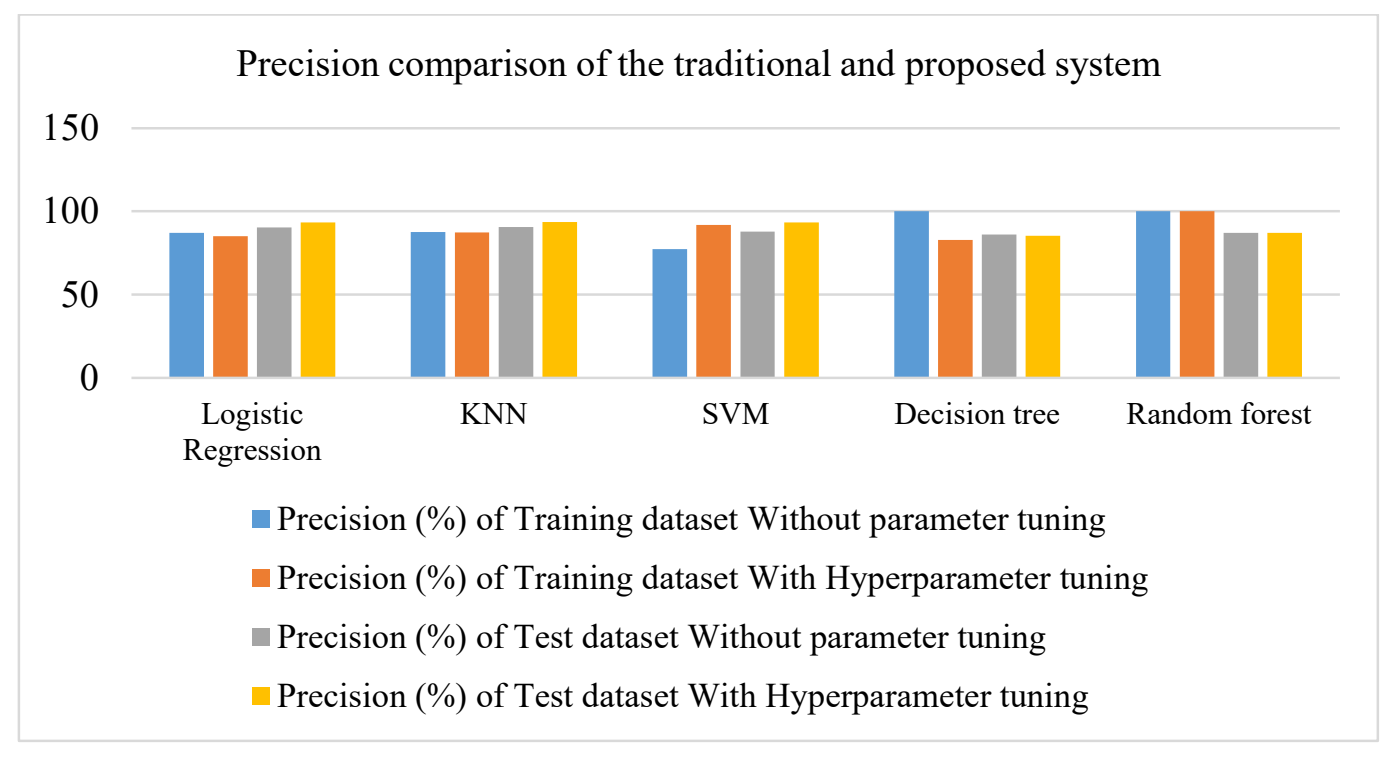

Figure 9. The graphical comparison of precision.

Table 6. Comparison of recall

\begin{tabular}{|c|c|c|c|c|}
\hline \multirow{2}{*}{$\begin{array}{c}\text { Machine } \\
\text { learning } \\
\text { algorithms }\end{array}$} & \multicolumn{2}{|c|}{ Recall (\%) of Training dataset } & \multicolumn{2}{c|}{ Recall (\%) of Test dataset } \\
\cline { 2 - 5 } & $\begin{array}{c}\text { Without } \\
\text { parameter } \\
\text { tuning }\end{array}$ & $\begin{array}{c}\text { With } \\
\text { Hyperparameter } \\
\text { tuning }\end{array}$ & $\begin{array}{c}\text { Without } \\
\text { parameter } \\
\text { tuning }\end{array}$ & $\begin{array}{c}\text { With } \\
\text { Hyperparameter } \\
\text { tuning }\end{array}$ \\
\hline LR & 90.98 & 89.47 & 87.50 & 87.50 \\
\hline KNN & 90.23 & 87.22 & 90.62 & 90.62 \\
\hline SVM & 94.74 & 94.74 & 90.62 & 87.50 \\
\hline DT & 100 & 90.98 & 78.12 & 90.62 \\
\hline RF & 100 & 100 & 84.38 & 84.38 \\
\hline
\end{tabular}

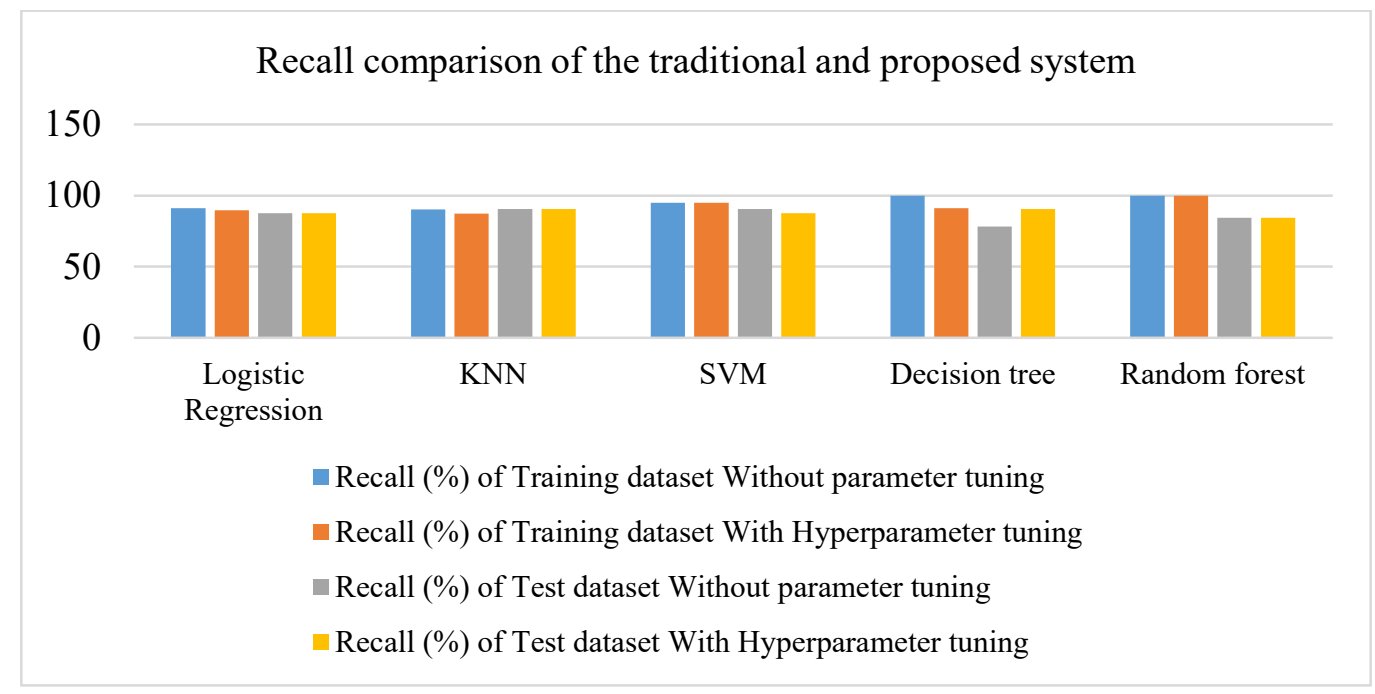

Figure 10. The graphical comparison of recall. 
Table 7. Comparison of F1 score

\begin{tabular}{|c|c|c|c|c|}
\hline $\begin{array}{c}\text { Machine } \\
\text { learning } \\
\text { algorithms }\end{array}$ & \multicolumn{2}{|c|}{ F1 score (\%) of Training dataset } & \multicolumn{2}{c|}{ F1 score (\%) of Test dataset } \\
\cline { 2 - 5 } & $\begin{array}{c}\text { Without } \\
\text { parameter } \\
\text { tuning }\end{array}$ & $\begin{array}{c}\text { With } \\
\text { Hyperparameter } \\
\text { tuning }\end{array}$ & $\begin{array}{c}\text { Without } \\
\text { parameter } \\
\text { tuning }\end{array}$ & $\begin{array}{c}\text { With } \\
\text { Hyperparameter } \\
\text { tuning }\end{array}$ \\
\hline LR & 88.97 & 87.18 & 88.89 & 90.32 \\
\hline KNN & 88.89 & 87.22 & 90.62 & 92.06 \\
\hline SVM & 85.14 & 93.33 & 89.23 & 90.32 \\
\hline DT & 100 & 86.74 & 81.97 & 87.88 \\
\hline RF & 100 & 100 & 85.71 & 85.71 \\
\hline
\end{tabular}

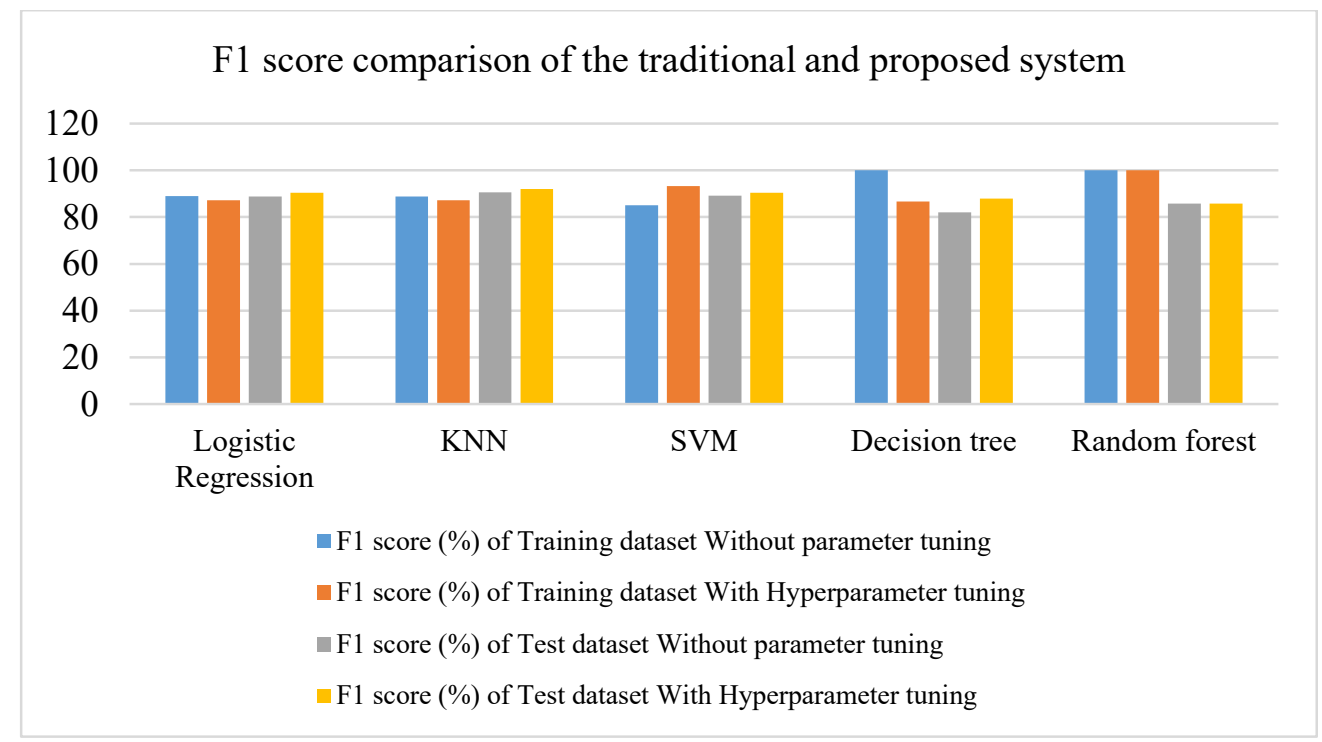

Figure 11. The graphical comparison of F1 score.

Table 4, Table 5, Table 6, Table 7, Figure 8, Figure 9, Figure 10, and Figure 11 represent the performance comparison between without and with the hyperparameters tuning approach of five machine learning algorithms in terms of accuracy, precision, recall, and F1 score. These comparisons show that the prediction systems with hyperparameters tuning provide better results than traditional prediction systems.

\section{Conclusion}

In this paper, the traditional and proposed system was implemented to predict Cleveland heart disease dataset. In both cases, machine learning algorithms include Logistic Regression, K nearest neighbor, Support vector machine, Decision tree, Random Forest are used in the generating of the heart disease prediction model. These models mainly include five key stages, but the proposed model differs from the traditional system in terms of tuning hyperparameters. Whereas, without hyperparameters tuning, the LR, KNN, SVM, DT, and RF classifiers provide an accuracy rate of $88.52 \%, 90.16 \%$, $88.52 \%, 81.97 \%$, and $85.25 \%$ respectively. However, with the hyperparameters tuning approach, the LR, KNN, SVM, DT, and RF classifiers in the refined set takes the accuracy rate $90.16 \%, 91.80 \%$, 
$90.16 \%, 86.89 \%$, and $85.25 \%$ respectively. Hence, experimental results of performance evaluation on the heart dataset, it is concluded that the proposed model is more efficient, and it can improve the prediction of heart disease. The future aspect of this research will be implemented the model with the feature selection approach using different optimization techniques.

\section{References}

[1] Li, J. P., Haq, A. U., Din, S. U., Khan, J., Khan, A., \& Saboor, A. (2020). Heart Disease Identification Method Using Machine Learning Classification in E-Healthcare. IEEE Access, Vol. 8, 107562107582.

[2] Ali, L., Niamat, A., Khan, J. A., Golilarz, N. A., Xingzhong, X., Noor, A., \& Bukhari, S. A. C. (2019). An optimized stacked support vector machines based expert system for the effective prediction of heart failure. IEEE Access, Vol.7, 54007-54014.

[3] Beunza, J. J., Puertas, E., García-Ovejero, E., Villalba, G., Condes, E., Koleva, G., \& Landecho, M. F. (2019). Comparison of machine learning algorithms for clinical event prediction (risk of coronary heart disease). Journal of biomedical informatics, Vol.97, 103257.

[4] Motarwar, P., Duraphe, A., Suganya, G., \& Premalatha, M. (2020, February). Cognitive Approach for Heart Disease Prediction using Machine Learning. In 2020 International Conference on Emerging Trends in Information Technology and Engineering (ic-ETITE)IEEE, 1-5

[5] Mantovani, R. G., Horváth, T., Cerri, R., Vanschoren, J., \& de Carvalho, A. C. (2016, October). Hyperparameter tuning of a decision tree induction algorithm. In 2016 5th Brazilian Conference on Intelligent Systems (BRACIS) IEEE, 37-42

[6] Ali, A. A. (2019). Stroke Prediction using Distributed Machine Learning Based on Apache Spark. Stroke, Vol.28, No.15, 89-97.

[7] Chandrasegar, T., \& Choudhary, A. (2019, March). Heart Disease Diagnosis using a Machine Learning Algorithm. In 2019 Innovations in Power and Advanced Computing Technologies (i-PACT), IEEE, Vol. 1, 1-4).

[8] Latha, C. B. C., \& Jeeva, S. C. (2019). Improving the accuracy of prediction of heart disease risk based on ensemble classification techniques. Informatics in Medicine Unlocked, Vol.16, 100203.

[9] Singh, D., \& Samagh, J. S. (2020). A COMPREHENSIVE REVIEW OF HEART DISEASE PREDICTION USING MACHINE LEARNING. Journal of Critical Reviews, Vol.7, No.12, 2020.

[10] Ayon, S. I., Islam, M. M., \& Hossain, M. R. (2020). Coronary artery heart disease prediction: a comparative study of computational intelligence techniques. IETE Journal of Research, 1-20.

[11] Ahmed, H., Younis, E. M., Hendawi, A., \& Ali, A. A. (2020). Heart disease identification from patients' social posts, machine learning solution on Spark. Future Generation Computer Systems, Vol.111, 714-722.

[12] Alizadehsani, R., Abdar, M., Roshanzamir, M., Khosravi, A., Kebria, P. M., Khozeimeh, F., \& Acharya, U. R. (2019). Machine learning-based coronary artery disease diagnosis: A comprehensive review. Computers in Biology and Medicine, Vol.111, 103346.

[13] Amin, M. S., Chiam, Y. K., \& Varathan, K. D. (2019). Identification of significant features and data mining techniques in predicting heart disease. Telematics and Informatics, 36, 82-93.

[14] Bashir, S., Khan, Z. S., Khan, F. H., Anjum, A., \& Bashir, K. (2019, January). Improving heart disease prediction using feature selection approaches. In 2019 16th International Bhurban Conference on Applied Sciences and Technology (IBCAST), IEEE, 619-623

[15] Mohan, S., Thirumalai, C., \& Srivastava, G. (2019). Effective heart disease prediction using hybrid machine learning techniques. IEEE Access, 7, 81542-81554. 
[16] Wu, C. S. M., Badshah, M., \& Bhagwat, V. (2019, July). Heart Disease Prediction Using Data Mining Techniques. In Proceedings of the 2019 2nd International Conference on Data Science and Information Technology, 7-11.

[17] Reddy, N. S. C., Nee, S. S., Min, L. Z., \& Ying, C. X. (2019). Classification and feature selection approaches by machine learning techniques: Heart disease prediction. International Journal of Innovative Computing, Vol.9, No.1.

[18] Ziasabounchi, N., \& Askerzade, I. (2014). ANFIS based classification model for heart disease prediction. International Journal of Electrical \& Computer Sciences IJECS-IJENS, Vol.14, No.02, 712.

[19] Haq, A. U., Li, J. P., Memon, M. H., Nazir, S., \& Sun, R. (2018). A hybrid intelligent system framework for the prediction of heart disease using machine learning algorithms. Mobile Information Systems, 2018.

[20] Fitriyani, N. L., Syafrudin, M., Alfian, G., \& Rhee, J. (2020). HDPM: An Effective Heart Disease Prediction Model for a Clinical Decision Support System. IEEE Access, 8, 133034-133050. 